\title{
Effects of endoplasmic reticulum stress on apoptosis induction in radioresistant macrophages
}

\author{
HIRONORI YOSHINO $^{1}$, YU KUMAI ${ }^{2}$ and IKUO KASHIWAKURA ${ }^{1}$ \\ ${ }^{1}$ Department of Radiation Science, Hirosaki University Graduate School of Health Sciences; \\ ${ }^{2}$ Department of Radiological Technology, Hirosaki University School of Health Sciences, Hirosaki, Aomori 036-8564, Japan
}

Received January 25, 2016; Accepted January 19, 2017

DOI: $10.3892 / \mathrm{mmr} .2017 .6298$

\begin{abstract}
Macrophages are important in the host's immune defense against pathogens. However, recent evidence has demonstrated that macrophages are also involved in the development of disease, including cancer. Therefore, it is important to regulate apoptosis in tumor-related macrophages for effective cancer treatment. In the present study, the effect of endoplasmic reticulum (ER) stress on apoptosis induction was examined in human monocytic cell-derived macrophages. Radiation therapy in cancer results in irradiating macrophages as well as cancer cells in the tumor microenvironment. Since ER stress has been demonstrated to sensitize cancer cells to radiation, it was hypothesized that ER stress may induce a similar effect in macrophages. Therefore, the effect of combination treatment with ER stress inducers and ionizing radiation on macrophage apoptosis was examined. Treatment of macrophages with ER stress inducers thapsigargin and tunicamycin, enhanced unfolded protein responses, including phosphorylation of eukaryotic initiation factor $2-\alpha$ and increased expression of binding immunoglobulin protein. Furthermore, treatment with thapsigargin and tunicamycin induced apoptosis in macrophages compared with untreated cells, although ionizing radiation did not. The thapsigargin-induced apoptosis in macrophages was demonstrated to be caspase-3-dependent. Finally, combination treatment with thapsigargin and ionizing
\end{abstract}

Correspondence to: Dr Hironori Yoshino, Department of Radiation Science, Hirosaki University Graduate School of Health Sciences, 66-1 Honcho, Hirosaki, Aomori 036-8564, Japan

E-mail: hyoshino@hirosaki-u.ac.jp

Abbreviations: BiP, binding immunoglobulin protein; DMSO, dimethyl sulfoxide; eIF2, eukaryotic initiation factor 2; ER, endoplasmic reticulum; FBS, fetal bovine serum; HRP, horseradish peroxidase; $\mathrm{PBS}(-), \mathrm{Ca}^{2+}$ and $\mathrm{Mg}^{2+}$ free phosphate-buffered saline; PARP, poly (ADP-ribose) polymerase; PI, propidium iodide; SERCA, sarco/endoplasmic reticulum $\mathrm{Ca}^{2+}$-ATPase; UPR, unfolded protein response; Z-VAD-FMK, carbobenzoxy-valyl-alanyl-aspartyl-[O-methyl]-fluoromethylketone

Key words: endoplasmic reticulum stress, macrophage, apoptosis, ionizing radiation, caspase radiation, did not result in any significant change in macrophage apoptosis. The present study demonstrated that ER stress regulated apoptosis in radioresistant macrophages and that ionizing radiation had no added effect on ER stress-induced apoptosis in macrophages.

\section{Introduction}

Macrophages are tissue-resident innate immune cells that are important in the host's defense against pathogens. Furthermore, macrophages are involved in maintaining tissue homeostasis and promoting wound healing of injured tissues (1). However, recent evidence has demonstrated that macrophages are also involved in many diseases, including obesity, diabetes, and cancer (2). In cancer, macrophages that reside in the tumor microenvironment are referred to as tumor-associated macrophages, and are associated with poor therapeutic efficacy because they enable drug resistance in cancer cells and promote tumor growth, immunosuppression and metastasis (3-5). Therefore, the development of strategies to selectively regulate tumor-associated macrophages may result in more effective cancer treatments.

Radiation therapy is extensively used for various cancers. Ionizing radiation directly or indirectly causes DNA damage, leading to cell cycle arrest and death. In general, rapidly proliferating and undifferentiated cells are relatively sensitive to ionizing radiation, whereas non-proliferating and highly differentiated cells exhibit radioresistance. Consistently, although monocytes are sensitive to DNA damage by oxidative stress, including ionizing radiation, macrophages or dendritic cells, that are differentiated from monocytes, are resistant to DNA damage (6). Therefore, although radiation therapy can target the macrophages that reside in the tumor microenvironment as well as the cancer cells, macrophages are not killed by ionizing radiation. Furthermore, since activation of DNA damage response pathways, including tumor protein p53 (p53) (7), is very weak in macrophages compared with monocytes (6), cell death mechanisms that are independent of DNA damage and the p53 pathway are preferable to induce macrophage apoptosis as a therapeutic intervention.

Endoplasmic reticulum (ER) stress is induced by the accumulation of unfolded and/or misfolded proteins in the ER lumen $(8,9)$. To deal with these accumulated unfolded and/or misfolded proteins, cells activate the unfolded protein 
response (UPR) signaling pathway. UPR triggers the following three main cellular responses: i) inhibition of protein translation by phosphorylating eukaryotic initiation factor 2- $\alpha$ $(\mathrm{eIF} 2 \alpha)$, ii) expression of ER chaperone proteins such as binding immunoglobulin protein (BiP), and iii) degradation of unfolded and/or misfolded proteins in the ER $(8,9)$. When cells are exposed to excessive and/or prolonged ER stress, apoptotic pathways are initiated in both p53-dependent and independent manners (10). Therefore, ER stress inducers may cause apoptosis in macrophages. Furthermore, because previous studies have demonstrated that ER stress sensitizes cancer cells to radiation $(11,12)$, combination treatment with an ER stress inducer and ionizing radiation may effectively induce apoptosis in macrophages. However, the synergistic effects of ER stress and ionizing radiation on apoptosis induction in macrophages remain unknown. Therefore, in the present study, the effects of chemical ER stress inducers thapsigargin and tunicamycin were examined on the UPR pathway and apoptosis in THP-1-derived macrophage-like cells. Furthermore, the effect of combination treatment with ER stress inducers and ionizing radiation was examined on macrophage apoptosis induction.

\section{Materials and methods}

Reagents. Phorbol 12-myristate 13-acetate (PMA), dimethyl sulfoxide(DMSO), propidiumiodide(PI), and thapsigargin were purchased from Sigma-Aldrich; Merck Millipore (Darmstadt, Germany). Tunicamycin was purchased from Enzo Life Sciences (Farmingdale, NY, USA). Carbobenzoxy- valyl-alany l-aspartyl-[O-methyl]-fluoromethylketone (Z-VAD-FMK) was purchased from Peptide Institute, Inc. (Osaka, Japan). Primary antibodies targeting phosphorylated (p-) eIF2 $\alpha$ (Ser51; cat. no. 3597), BiP (cat. no. 3177), caspase-3 (cat. no. 9662), poly (ADP-ribose) polymerase (PARP; cat. no. 9532), and $\beta$-actin (cat. no. 4967), and secondary anti-rabbit immunoglobulin (Ig) G horseradish peroxidase (HRP)-linked antibody (cat. no. 7074) and anti-mouse IgG HRP-linked antibody (cat. no. 7076) were purchased from Cell Signaling Technology Japan, K.K. (Tokyo, Japan).

Cell culture and treatments. Since the THP-1 human acute monocytic leukemia cell line is negative for $\mathrm{p} 53$ expression due to a 26 bp deletion beginning at codon 174 of the p53 coding sequence (13), THP-1-derived macrophage-like cells are suitable for exploring apoptosis in macrophages independent of the p53 pathway. THP-1 human acute monocytic leukemia cells were obtained from RIKEN BioResource Center (Tsukuba, Japan). The cells were cultured in Gibco RPMI1640 (Thermo Fisher Scientific, Inc., Waltham, MA, USA) supplemented with $1 \%$ penicillin and streptomycin and $10 \%$ heat-inactivated fetal bovine serum (Japan Bio Serum Co., Ltd., Nagoya, Japan) at $37^{\circ} \mathrm{C}$ in a humidified atmosphere containing $5 \%$ $\mathrm{CO}_{2}$. THP-1-derived macrophages (hereafter termed macrophages) were prepared as previously described (14). THP-1 cells $\left(2 \times 10^{5}\right.$ cells $\left./ \mathrm{ml}\right)$ were plated in $35-\mathrm{mm}$ dishes with $2 \mathrm{ml}$ of medium containing $100 \mathrm{ng} / \mathrm{ml}$ PMA and cultured for $48 \mathrm{~h}$, then the medium was replaced with fresh PMA-free medium, and the macrophage-like cells were used in experiments.

For the treatments, THP- 1 cells $\left(2 \times 10^{5}\right.$ cells/dish) or macrophages were cultured in the presence of either 50 or $500 \mathrm{nM}$ thapsigargin, or 5 or $20 \mu \mathrm{g} / \mathrm{ml}$ tunicamycin for the indicated time periods. Following treatment, cells were harvested by trypsinization followed by centrifugation $\left(300 \times g\right.$ at $4^{\circ} \mathrm{C}$ for $5 \mathrm{~min})$ for further analysis. Z-VAD-FMK $(50 \mu \mathrm{M})$, a general caspase inhibitor, was added $1 \mathrm{~h}$ prior to thapsigargin treatment. Irradiation (10-Gy) was performed at $1 \mathrm{~h}$ following thapsigargin treatment. Since thapsigargin, tunicamycin, and Z-VAD-FMK were dissolved in DMSO, DMSO was used as vehicle control.

In vitro $X$-ray irradiation. X-ray irradiation $(150 \mathrm{kVp}, 20 \mathrm{~mA}$, $0.5 \mathrm{~mm} \mathrm{Al}$, and $0.3-\mathrm{mm} \mathrm{Cu}$ filters) was performed using an MBR-1520R-3 X-ray generator (Hitachi, Ltd., Tokyo, Japan) at a distance of $45 \mathrm{~cm}$ from the focus, with a dose rate of $1.02-1.05 \mathrm{~Gy} / \mathrm{min}$.

Cell cycle analysis. Harvested cells were fixed with $70 \%$ ethanol overnight at $-20^{\circ} \mathrm{C}$. Fixed cells were washed with $\mathrm{Ca}^{2+}$ - and $\mathrm{Mg}^{2+}$-free phosphate-buffered saline [PBS(-)] and then treated with RNase A $(200 \mu \mathrm{g} / \mathrm{ml})$ at $37^{\circ} \mathrm{C}$ for $30 \mathrm{~min}$ to hydrolyze RNA. Following treatment, cells were washed with PBS(-) and stained with propidium iodide (PI; $30 \mu \mathrm{g} / \mathrm{ml}$ ) for $30 \mathrm{~min}$ in the dark. Finally, the cells were filtered with a $40 \mu \mathrm{m}$ cell strainer (BD Biosciences, Franklin Lakes, NJ, USA) and cell cycle distribution was analyzed by flow cytometry (Cytomics FC500 with CXP software ver. 2; Beckman Coulter, Inc., Brea, CA, USA).

SDS-PAGE and western blotting. Harvested cells were lysed in 1X Laemmli Sample Buffer (Bio-Rad Laboratories, Inc., Hercules, CA, USA) containing 2.5\% 2-mercaptoethanol, by sonication and boiling for $10 \mathrm{~min}$. Protein concentration was determined using the XL-Bradford assay kit (APRO Science, Tokushima, Japan) and a SmartSpec Plus spectrophotometer (Bio-Rad Laboratories, Inc.). The proteins ( $\sim 5 \mu \mathrm{g} /$ lane) were separated using 4-20\% Mini-PROTEAN TGX precast gels (Bio-Rad Laboratories, Inc.) and were transferred onto polyvinylidene difluoride (PVDF) membranes from Trans-Blot Turbo Mini PVDF Transfer Packs (Bio-Rad Laboratories, Inc.) using the Trans-Blot Turbo transfer system (Bio-Rad Laboratories, Inc.). Membranes were blocked in TBST buffer (10 mM HCl, pH 7.5, $100 \mathrm{mM} \mathrm{NaCl}$, and $0.1 \%$ Tween-20) that contained 5\% non-fat milk or PVDF Blocking Reagent for Can Get Signal (Toyobo Co., Ltd., Osaka, Japan) at room temperature for $1 \mathrm{~h}$. The membranes were probed with each primary antibody in the TBST buffer containing 5\% non-fat skim milk or Can Get Signal immunoreaction enhancer solution 1 (Toyobo Co., Ltd.) overnight at $4^{\circ} \mathrm{C}$. The following primary antibodies were used: Anti-p-eIF $2 \alpha$ antibody (1:4,000), anti-BIP antibody (1:4,000), anti-caspase-3 antibody $(1: 3,000)$, anti-PARP antibody $(1: 3,000)$, or anti-actin antibody $(1: 4,000)$. The membranes were then incubated with HRP-conjugated secondary antibodies in TBST buffer containing 5\% non-fat milk or Can Get Signal immunoreaction enhancer solution 2 (Toyobo Co., Ltd.) for $1 \mathrm{~h}$. The following secondary antibodies were used: HRP-linked anti-rabbit IgG antibody (1:10,000) or HRP-linked anti-mouse IgG antibody $(1: 10,000)$. Antigens were visualized using the Clarity enhanced chemiluminescence western blotting substrate (Bio-Rad Laboratories, Inc.). Blot stripping was 
performed using Stripping Solution (Wako Pure Chemical Industries, Ltd., Osaka, Japan).

Statistical analysis. Data are presented as the mean \pm standard error. Comparisons between the control and experimental groups were performed using a two-sided Student's $t$-test. $\mathrm{P}<0.05$ was considered to indicate a statistically significant difference. Excel 2010 (Microsoft Corporation, Redmond, WA, USA) with the add-in Statcel 3 software (The Publisher OMS Ltd., Tokyo, Japan) was used to perform statistical analyses.

\section{Results}

ER stress-related protein expression in macrophages treated with ER stress inducers. The effects of ER stress inducers thapsigargin and tunicamycin were investigated in THP-1-derived macrophages. As demonstrated in Fig. 1A, expression levels of the ER stress protein markers p-eIF $2 \alpha$ and BiP were visibly increased in macrophages following treatment with thapsigargin and tunicamycin for $24 \mathrm{~h}$, compared with macrophages treated with DMSO vehicle control. This finding suggests that macrophages responded to ER stress. Ionizing radiation has been reported to induce ER stress in cancer cells $(15,16)$, therefore the expression levels of p-eIF $2 \alpha$ and BiP were examined in macrophages following X-ray irradiation. No change was observed in p-eIF $2 \alpha$ and BiP protein expression in irradiated compared with untreated cells, suggesting that X-ray irradiation does not induce ER stress in macrophages (Fig. 1B).

ER stress inducers effect on apoptosis. The effect of X-ray irradiation and ER stress induction on apoptosis was then examined in THP-1 cells and THP-1-derived macrophages, by cell cycle phase distribution analysis using flow cytometry. As demonstrated in Fig. 2A, X-ray irradiation did not increase the $\%$ of macrophages in the sub-G1 fraction, which is a hallmark of apoptosis, compared with untreated macrophages. However, when the immature monocytic THP-1 cells were irradiated, a significant increase in the $\%$ of cells in the sub-G1 fraction was observed compared to untreated THP-1 cells (Fig. 2A). These data suggest that the differentiation status of macrophages renders them resistant to ionizing radiation. By contrast, treatment with ER stress inducers thapsigargin and tunicamycin significantly induced the $\%$ of cells in the sub-G1 fraction in both macrophages and THP-1 cells, compared to their respective untreated cell control group (Fig. 2B and C). Finally, thapsigargin exhibited a more potent effect in inducing apoptosis in macrophages than tunicamycin, as exhibited by markedly higher numbers of macrophages in the sub-G1 fraction following $72 \mathrm{~h}$ of thapsigargin treatment compared with $72 \mathrm{~h}$ of tunicamycin treatment (Fig. 2B and C).

Involvement of caspases in thapsigargin-induced apoptosis. Caspase-3 is a key effector protease that is responsible for DNA fragmentation during apoptosis. Therefore, the hypothesis that thapsigargin-induced apoptosis may be mediated by caspase- 3 activation was further examined. As demonstrated in Fig. 3A, treatment of macrophages with $500 \mathrm{nM}$ thapsigargin induced caspase-3 cleavage, compared with macrophages treated with DMSO vehicle control. Furthermore, increased cleavage of PARP, which is a target of cleaved caspase-3,
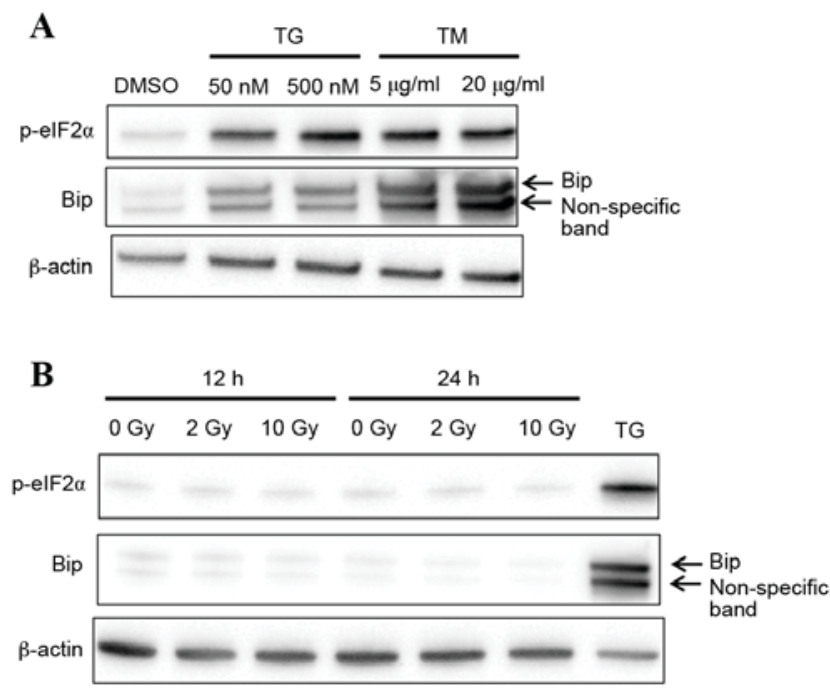

Figure 1. ER stress-related protein expression in THP-1-derived macrophages. (A) THP-1-derived macrophages were treated with 50 or $500 \mathrm{nM}$ TG or 5 or $20 \mu \mathrm{g} / \mathrm{ml} \mathrm{TM}$ for $24 \mathrm{~h}$. Cells treated with DMSO vehicle were used as control. (B) X-irradiated macrophages ( 2 or $10 \mathrm{~Gy}$ ) were cultured for 12 or $24 \mathrm{~h}$. Non-irradiated cells were used as a negative control and cells treated with $500 \mathrm{nM}$ TG for $24 \mathrm{~h}$ were used as a positive control. Expression levels of p-eIF $2 \alpha$ and BiP were analyzed by western blotting, with $\beta$-actin as a loading control. ER, endoplasmic reticulum; TG, thapsigargin; TM, tunicamycin; DMSO, dimethyl sulfoxide; Gy, gray (unit); p-eIF2 $\alpha$, phosphorylated eukaryotic initiation factor $2-\alpha$; BiP, binding immunoglobulin protein.

was observed in thapsigargin-treated macrophages compared with DMSO-treated macrophages (Fig. 3B). Finally, thapsigargin-induced apoptosis in macrophages was demonstrated to be caspase-dependent, as treatment with the pan-caspase inhibitor Z-VAD-FMK effectively prevented PARP cleavage and reduced the $\%$ of cells in the sub-G1 fraction in thapsigargin-treated macrophages (Fig. 3B and C). Taken together, these results suggest that thapsigargin induces caspase-mediated apoptosis.

Effect of combination treatment in macrophages with thapsigargin and $X$-ray irradiation. Previous studies have demonstrated that ER stress causes radiosensitization in cancer cells $(11,12)$. Therefore, the hypothesis that combination treatment with thapsigargin and X-ray irradiation may enhance apoptosis in THP-1-derived macrophages was examined. Treatment with thapsigargin for $48 \mathrm{~h}$ induced apoptosis in macrophages, however, combination treatment with 10-Gy irradiation did not affect the $\%$ of apoptotic cells compared with thapsigargin treatment alone (Fig. 4). Similarly, combination treatment of macrophages with tunicamycin and 10-Gy irradiation had no effect on apoptosis rate compared with treatment with tunicamycin alone (data not shown). These data suggest that there is no synergistic effect of ER inducers and X-ray irradiation on apoptosis of THP-1 derived macrophages.

\section{Discussion}

Although macrophages are important in the host's immune defense against pathogens, they are also associated with many diseases, including cancer (2). Therefore, it is crucial for effective cancer treatment to regulate macrophage apoptosis. 

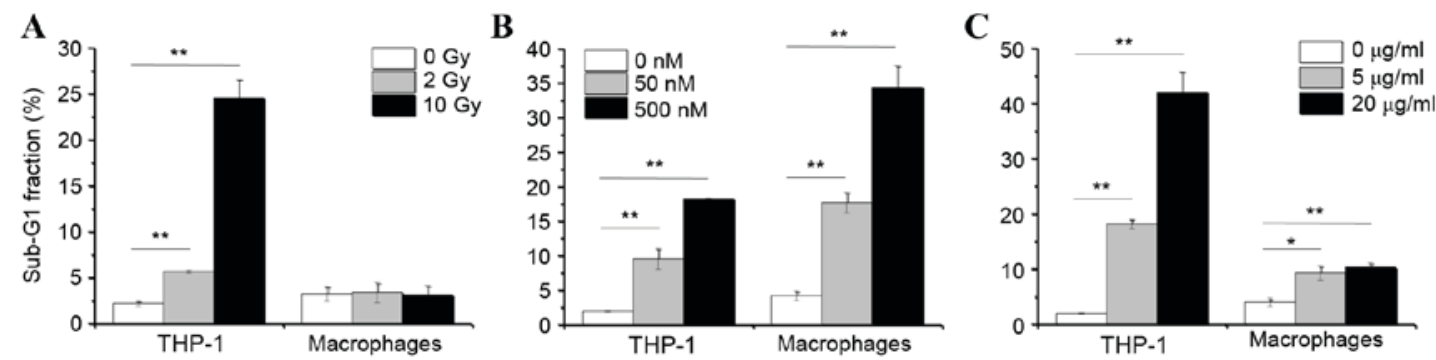

Figure 2. Effects of X-ray irradiation and ER stress inducers on apoptosis. THP-1 cells and THP-1-derived macrophages were cultured for 48 and $72 \mathrm{~h}$ respectively, following (A) irradiation with 0,2 or $10 \mathrm{~Gy}$, (B) treatment with 0,50 or $500 \mathrm{nM} \mathrm{TG}$ or (C) treatment with $0,5 \mathrm{or} 20 \mu \mathrm{g} / \mathrm{ml} \mathrm{TM}$. Following treatments, cells were analyzed for cell cycle distribution by flow cytometry. Data are presented as the mean \% of cells in the Sub-G1 fraction \pm standard error of 3 independent experiments. ${ }^{*} \mathrm{P}<0.05$ and $^{* *} \mathrm{P}<0.01$ with comparisons indicated by lines. ER, endoplasmic reticulum; TG, thapsigargin; TM, tunicamycin; Gy, gray (unit).

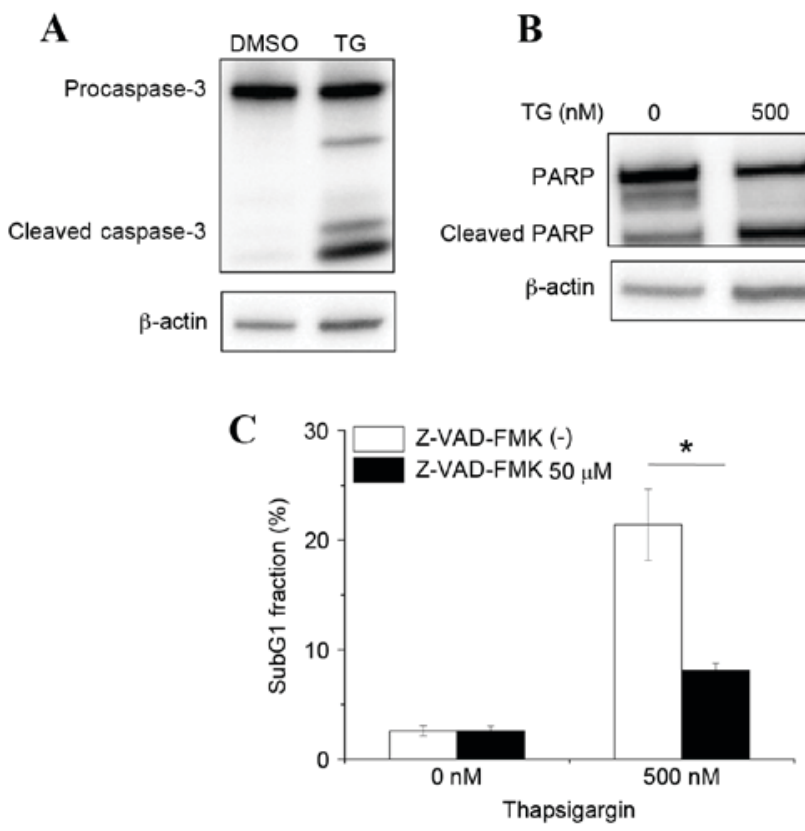

Figure 3. Involvement of caspase-3 in TG-induced apoptosis. (A) THP-1-derived macrophages were treated with $500 \mathrm{nM}$ TG for $48 \mathrm{~h}$ and caspase-3 cleavage was examined by western blotting. $\beta$-actin was used as a loading control. (B) THP-1-derived macrophages were pretreated with $50 \mu \mathrm{M} \mathrm{Z-VAD-FMK} \mathrm{for} 1 \mathrm{~h}$, then treated with $500 \mathrm{nM}$ TG for $48 \mathrm{~h}$. PARP cleavage was examined by western blotting, with $\beta$-actin as a loading control. (C) Macrophages were treated as in panel B, and apoptosis was examined by flow cytometry. Data are presented as the mean \% of cells in the Sub-G1 fraction \pm standard error of 3 independent experiments. "P<0.05, with comparisons indicated by lines. DMSO, dimethyl sulfoxide; Z-VAD-FMK, carbobenzoxy-valyl-alanyl-aspartyl-[O-methyl] -fluoromethylketone; PARP, poly (ADP-ribose) polymerase; TG, thapsigargin.

In the present study, the potential of ER stress as a regulator of apoptosis in radioresistant macrophages was explored. The present findings demonstrated that radioresistant macrophages responded to ER stress inducers, leading to enhanced apoptosis through caspase activation in a p53-independent manner. Consistent with the present results, mouse macrophage-like RAW 264.7 cells that are treated with lipopolysaccharide and interferon- $\gamma$, undergo apoptosis through ER stress-mediated signaling pathways without the accumulation of $\mathrm{p} 53$ protein (17). Furthermore, free cholesterol induces ER stress-mediated apoptosis in mouse macrophages (18). Therefore, it is considered that ER stress is a promising target pathway to regulate macrophage apoptosis.

In the present study, two types of ER stress-inducing agents were used: Thapsigargin and tunicamycin. Thapsigargin induces ER stress by irreversibly inhibiting the sarco/endoplasmic reticulum $\mathrm{Ca}^{2+}$-ATPase (SERCA), while tunicamycin

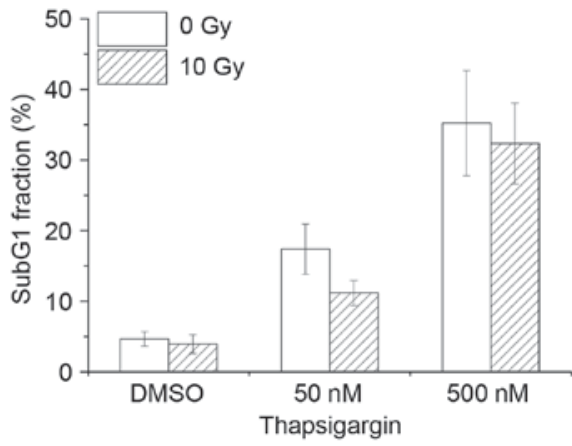

Figure 4. Effect of combination treatment with thapsigargin and X-ray irradiation in macrophage apoptosis. THP-1-derived macrophages were pretreated with 50 or $500 \mathrm{nM}$ thapsigargin for $1 \mathrm{~h}$, then exposed to $10 \mathrm{~Gy}$ irradiation. Cells that were treated with DMSO vehicle and cells that were not treated with irradiation were used as negative controls. Following $72 \mathrm{~h}$ culture post-irradiation, apoptosis was examined by flow cytometry. Data are presented as the mean \% of cells in the Sub-G1 fraction \pm standard error of 3 independent experiments. DMSO, dimethyl sulfoxide; Gy, gray (unit). 
inhibits N-linked glycosylation (19-21). The present study demonstrated that thapsigargin was more effective in inducing apoptosis in radioresistant macrophages than tunicamycin. SERCA pumps are important in maintaining low free cytosolic $\mathrm{Ca}^{2+}$ levels, and their inhibition activates the $\mathrm{Ca}^{2+}$-mediated apoptotic pathways (22). Buckley and Whorton (23) have reported that tunicamycin, as well as thapsigargin, induces $\mathrm{Ca}^{2+}$ release from the intracellular $\mathrm{Ca}^{2+}$ stores in bovine aortic endothelial cells. They demonstrated that partial refilling of the intracellular $\mathrm{Ca}^{2+}$ stores occurred in the cells treated with tunicamycin in the presence of extracellular $\mathrm{Ca}^{2+}$, whereas this effect was not observed in the cells treated with thapsigargin. This result indicates that endoplasmic reticulum $\mathrm{Ca}^{2+}$-ATPase is active following tunicamycin treatment. Therefore, it is thought that the inhibition of SERCA pumps may be an attractive strategy for regulating macrophage apoptosis.

Ionizing radiation induces ER stress in both normal and cancer cells $(15,16,24)$. For example, ionizing radiation increases BiP mRNA expression levels in the IEC-6 rat small intestinal epithelium cell line (16) and eIF2 $\alpha$ phosphorylation in the MDA-MB-231 human breast cancer cell line $(15,24)$. In the present study, expression levels of p-eIF2 $\alpha$ and BiP in macrophages were not affected by ionizing radiation, suggesting that radiation-induced ER stress depends on cell type. IEC-6 and MDA-MB-231 cells demonstrate apoptotic features following ionizing radiation $(16,24)$, whereas the present study demonstrated that irradiated macrophages did not undergo apoptosis. Therefore, there may be an association between the sensitivity of cells to radiation-induced ER stress and radiation-induced apoptosis.

Tunicamycin or other ER stress inducers enhance the radiosensitivity of cancer cells $(11,12,24,25)$. Furthermore, Lee et al (16) have reported that tunicamycin and thapsigargin enhance ionizing radiation-induced caspase-3 activation in IEC- 6 cells. Therefore, the hypothesis that combination treatment of ER stress inducers and ionizing radiation may kill macrophages in the tumor microenvironment was examined. In the present study, no synergistic effect of ER stress inducers and irradiation on apoptosis induction in macrophages was observed. Yamamori et al (12) demonstrated that ER stress sensitizes cells to radiation by impairing DNA double-strand break repair through degradation of the DNA repair protein RAD51 recombinase. Because p53 is important in apoptosis induction following DNA damage, synergistic effects of ER stress and X-ray irradiation might not be feasible in macrophages derived from p53-negative THP-1 cells. However, considering that ionizing radiation did not attenuate ER stress-induced apoptosis in macrophages (Fig. 4), combination treatment of ER stress inducers with radiotherapy may still be an effective cancer therapy due to its synergistic effect on cancer cell radiosensitization.

In conclusion, the present study demonstrated that ER stress induced apoptosis in radioresistant macrophages. In the present study, macrophages were produced by differentiation of monocytic THP-1 cells by PMA stimulation, allowing investigations of macrophage apoptosis mechanisms in a p53-independent background. However, there are many subsets of macrophages in vivo and their phenotype and function varies widely depending on the subset (1). Therefore, future studies will be needed to address in more detail whether ER stress induces apoptosis in disease-related macrophages, and in particular tumor-associated macrophages.

\section{Acknowledgements}

The present study was supported by a Hirosaki University Grant for Exploratory Research by Young Scientists and Newly Appointed Scientists. This work was also partially supported by JSPS KAKENHI, Grant-in-Aid for Scientific Research (grant no. 15K09985), a Grant for Hirosaki University Institutional Research, and the Takeda Science Foundation [HY]. The authors would like to thank Enago (www.enago.jp) for the English language review.

\section{References}

1. Murray PJ and Wynn TA: Protective and pathogenic functions of macrophage subsets. Nat Rev Immunol 11: 723-737, 2011.

2. Schultze JL, Schmieder A and Goerdt S: Macrophage activation in human diseases. Semin Immunol 27: 249-256, 2015.

3. Qian BZ and Pollard JW: Macrophage diversity enhances tumor progression and metastasis. Cell 141: 39-51, 2010.

4. Jinushi M, Chiba S, Yoshiyama H, Masutomi K, Kinoshita I, Dosaka-Akita H, Yagita H, Takaoka A and Tahara H: Tumor-associated macrophages regulate tumorigenicity and anticancer drug responses of cancer stem/initiating cells. Proc Natl Acad Sci USA 108: 12425-12430, 2011.

5. Ostuni R, Kratochvill F, Murray PJ and Natoli G: Macrophages and cancer: From mechanisms to therapeutic implications. Trends Immunol 36: 229-239, 2015.

6. Bauer M, Goldstein M, Christmann M, Becker H, Heylmann D and Kaina B: Human monocytes are severely impaired in base and DNA double-strand break repair that renders them vulnerable to oxidative stress. Proc Natl Acad Sci USA 108: 21105-21110, 2011.

7. Norbury CJ and Zhivotovsky B: DNA damage-induced apoptosis. Oncogene 23: 2797-2808, 2004.

8. Wu J and Kaufman RJ: From acute ER stress to physiological roles of the unfolded protein response. Cell Death Differ 13: 374-384, 2006.

9. Naidoo N: Cellular stress/the unfolded protein response: Relevance to sleep and sleep disorders. Sleep Med Rev 13: 195-204, 2009.

10. Li J, Lee B and Lee AS: Endoplasmic reticulum stress-induced apoptosis: Multiple pathways and activation of p53-up-regulated modulator of apoptosis (PUMA) and NOXA by p53. J Biol Chem 281: 7260-7270, 2006.

11. Contessa JN, Bhojani MS, Freeze HH, Rehemtulla A and Lawrence TS: Inhibition of N-linked glycosylation disrupts receptor tyrosine kinase signaling in tumor cells. Cancer Res 68: 3803-3809, 2008.

12. Yamamori T, Meike S, Nagane M, Yasui H and Inanami O: ER stress suppresses DNA double-strand break repair and sensitizes tumor cells to ionizing radiation by stimulating proteasomal degradation of Rad51. FEBS Lett 587: 3348-3353, 2013.

13. Sugimoto K, Toyoshima H, Sakai R, Miyagawa K, Hagiwara K, Ishikawa F, Takaku F, Yazaki Y and Hirai H: Frequent mutations in the 53 gene in human myeloid leukemia cell lines. Blood 79: 2378-2383, 1992.

14. Yoshino H, Saitoh T, Kozakai M and Kashiwakura I: Effects of ionizing radiation on retinoic acid-inducible gene-I-like receptors. Biomed Rep 3: 59-62, 2015.

15. Nagelkerke A, Bussink J, van der Kogel AJ, Sweep FC and Span PN: The PERK/ATF4/LAMP3-arm of the unfolded protein response affects radioresistance by interfering with the DNA damage response. Radiother Oncol 108: 415-421, 2013.

16. Lee ES, Lee HJ, Lee YJ, Jeong JH, Kang S and Lim YB: Chemical chaperones reduce ionizing radiation-induced endoplasmic reticulum stress and cell death in IEC- 6 cells. Biochem Biophys Res Commun 450: 1005-1009, 2014.

17. Gotoh T, Oyadomari S, Mori K and Mori M: Nitric oxide-induced apoptosis in RAW 264.7 macrophages is mediated by endoplasmic reticulum stress pathway involving ATF6 and CHOP. J Biol Chem 277: 12343-12350, 2002. 
18. Devries-Seimon T, Li Y, Yao PM, Stone E, Wang Y, Davis RJ, Flavell R and Tabas I: Cholesterol-induced macrophage apoptosis requires ER stress pathways and engagement of the type A scavenger receptor. J Cell Biol 171: 61-73, 2005.

19. Sagara Y and Inesi G: Inhibition of the sarcoplasmic reticulum $\mathrm{Ca} 2+$ transport ATPase by thapsigargin at subnanomolar concentrations. J Biol Chem 266: 13503-13506, 1991.

20. Lytton J, Westlin M and Hanley MR: Thapsigargin inhibits the sarcoplasmic or endoplasmic reticulum Ca-ATPase family of calcium pumps. J Biol Chem 266: 17067-17071, 1991.

21. Duksin D and Mahoney WC: Relationship of the structure and biological activity of the natural homologues of tunicamycin. J Biol Chem 257: 3105-3109, 1982

22. Dubois C, Prevarskaya N and Vanden Abeele F: The calcium-signaling toolkit: Updates needed. Biochim Biophys Acta 1863: 1337-1343, 2016.
23. Buckley BJ and Whorton AR: Tunicamycin increases intracellular calcium levels in bovine aortic endothelial cells. Am J Physiol 273: C1298-C1305, 1997.

24. Suzuki K, Gerelchuluun A, Hong Z, Sun L, Zenkoh J, Moritake T and Tsuboi K: Celecoxib enhances radiosensitivity of hypoxic glioblastoma cells through endoplasmic reticulum stress. Neuro Oncol 15: 1186-1199, 2013.

25. Yasui H, Takeuchi R, Nagane M, Meike S, Nakamura Y, Yamamori T, Ikenaka Y, Kon Y, Murotani H, Oishi M, et al: Radiosensitization of tumor cells through endoplasmic reticulum stress induced by PEGylated nanogel containing gold nanoparticles. Cancer Lett 347: 151-158, 2014. 